\title{
Teaching and Maintaining Quality of Resident Inpatient Progress Notes: Development of the Progress Note Quality Instrument
}

\author{
Carolyn Zyloney ${ }^{1}$, Joseph Modica ${ }^{2}$, Dimitrios Manou ${ }^{2}$, Christopher Mooney $^{2}$, and Robert \\ Thompson-Stone ${ }^{2}$ \\ ${ }^{1}$ Unity Hospital \\ ${ }^{2}$ University of Rochester Medical Center
}

May 5, 2020

\begin{abstract}
Rationale, aims and objectives: Since the widespread implementation of electronic medical records, there have been concerns about errors, excessive copying forward, and reduced quality in resident inpatient progress notes. The goal of this study was to create and validate a tool to assess the quality of progress notes written on an inpatient neurology service. Methods: A survey assessing perceptions of resident inpatient neurology progress note quality was administered to faculty and residents, and based on these results, a four item note assessment tool (PNQIv2) was developed. The tool assessed the following attributes: (1) accuracy, (2) synthesis, (3) focus, (4) patient-centeredness, and (5) copied-forward material. 120 note reviews were completed by 4 study investigators on 30 different resident progress notes utilizing 10 out of 16 possible residents (62.5\%). Mean PNQIv2 scores were calculated as well as inter-rater reliability for the overall PNQIv2 scores and their subsections using inter-class correlation coefficients and 95\% confidence intervals. Results: The PNQIv2 was found to have good inter-rater reliability at 0.7 and was considered quick and simple to use. The mean total PNQIv2 score was 9.2 (SD 2) out of 12 . $60 \%$ of notes were determined to be at least adequate quality by receiving a PNQIv2 score of [?] 9, and $63 \%$ of notes contained at least 2/3 copied-forward material. Conclusions: The investigators developed a progress note assessment tool that was simple and practical to use on the wards, with good inter-rater reliability, which may be useful to formally evaluate the quality of resident inpatient progress notes.
\end{abstract}

\section{Introduction}

Developing effective documentation and communication skills are key components of medical training. At most teaching hospitals, resident physicians are responsible for the majority of medical documentation including authorship of inpatient progress notes. The AAMC includes the ability to provide clear and succinct documentation as a core professional activity for individuals entering residency ${ }^{1}$. Due to the widespread implementation of electronic medical record (EMR) systems, providers and trainees need to learn how to optimally and efficiently document information in electronic systems while minimizing error. However, many medical schools and residency programs lack formal education on medical documentation and optimal use of EMR systems ${ }^{2,3,4,5}$.

There are several advantages to the use of an EMR including increased availability and legibility of records $s^{6,7,8}$. However, there have been unintended consequences including frequent use of copy forward functionality, excessive use of templates, increase in extraneous or redundant information in medical charts, and poor formatting of notes ${ }^{6,8-18}$. These factors can reduce the quality of notes and lead to errors in medical documentation, creating the need for measures to evaluate and teach optimal note-writing skills ${ }^{13,14,19.20,21}$.

Several prior studies have investigated ways to improve documentation and use of EMR as part of a medical curriculum $^{4,5,9,19,21-31}$. However, many of these studies have focused on medical billing and coding rather 
than on the overall quality of notes ${ }^{27-31}$. There has been prior research into developing and utilizing a note evaluation tool to formally assess medical documentation ${ }^{7,9,23,25,32,33}$, however the majority of these tools were lengthy with poorly defined anchors, thus rendering them impractical for use outside of a research setting. In one such study, an instrument called the PDQI-9 was used to evaluate admission, progress, and discharge notes at a teaching hospital ${ }^{9}$, and the authors noted that the PDQI-9 scores did not always correlate well to the reviewer's overall impression of the note. Another study evaluating use of the PDQI-9 tool to evaluate emergency department notes found that there was poor inter-rater reliability and that it was unable to reliably distinguish good notes from poor ones ${ }^{32}$. Another note assessment tool, the QNOTE, was used to evaluate primary care office notes ${ }^{33}$. There was good inter-rater reliability with the QNOTE, however the tool appeared to over-estimate the quality of notes when compared to a separate general impression score. Other studies used an assessment tool to evaluate whether certain components of the note (e.g. problem list or treatment plan) were present, but did not evaluate the quality of the note as a whole ${ }^{18,25}$.

In an effort to improve the quality of progress notes on the inpatient neurology service at the University of Rochester Medical Center (URMC), the investigators used existent literature to develop and implement a tool to objectively and comprehensively evaluate the quality of progress notes on the inpatient neurology service. The goal of the investigators was to create a tool that was both practical and simple to use on the neurology inpatient service and with the potential to expand its use to other inpatient services.

\section{Methods}

The present study was conducted at URMC between September 2016 and January 2019 and consisted of three phases: (1) a needs assessment survey of neurology residents and faculty; (2) a pilot phase to study the first iteration of a progress note quality instrument (PNQI), and; (3) a subsequent testing phase of a revised progress note quality instrument (PNQIv2). The protocol was reviewed by the University of Rochester's Human Subject Review Board and met criteria for exemption.

\section{The Neurology Inpatient Service}

On the inpatient neurology service at URMC, progress notes are predominantly written by junior residents (mostly PGY1) from the departments of psychiatry, anesthesia, medicine-pediatrics, and neurology who function in the capacity of the team intern. These junior residents rotate through the neurology service in 2-4 week blocks, completing a total of 2 to 8 weeks on the service throughout the academic year. All PGY1 residents are supervised by a senior neurology resident (PGY2 and PGY4) and an attending neurologist.

\section{Resident/Faculty Needs Assessment Survey}

In September 2016, a 10-item survey was administered to URMC neurology residents and faculty and recent residency graduates who worked on the inpatient adult neurology service within the past calendar year. Participation was voluntary and responses were collected anonymously via a web-based service. Respondents were asked to rate their perception of the quality of notes, including estimating the amount of copied forward material contained in the progress notes, the utility of the copy forward function and whether it created error. If respondents recalled having encountered errors in the notes, they were asked to identify the type of error.

\section{PNQI Pilot Phase}

Using the PDQI-9 as a model, the initial version of the PNQI was developed by the study investigators, to assess whether notes were: (1) up-to-date; (2) accurate; (3) relevant and focused, and; (4) comprehensive and thoughtful. Participants were asked to rate each domain using a 5-point scoring rubric with specific language-based descriptors. The scores from each of these categories were tabulated to formulate a total score ranging from 4 - 20, with higher scores representing higher quality notes. In addition, the PNQI evaluated the quantity of copied forward material from prior notes and whether the copied material created error.

During the 2017-2018 academic year, the investigators piloted the PNQI with junior residents rotating 
through the neurology service. Junior residents provided verbal consent to allow the study investigators to review their notes. Two members of the study staff (RTS, CZ) used the instrument to review progress notes written by 12 junior resident at the end of their two week block on the neurology service. Mean PNQI scores were calculated. To quantify inter-rater reliability for the overall PNQI scores and their subsections, we estimated using inter-class correlation coefficients (ICC) and 95\% confidence intervals (95\% CI) using a multi-level random effects mode (Stata/SE 14.2).

\section{PNQI Second Phase}

Based on results from the pilot phase, a revised version of the tool (PNQIv2) was created with the aim of improving inter-rater reliability and further enhancing usefulness (Figure 1). This version included four items assessing note attributes, however each attribute was scored on a three-point scale (total possible score of 12) rather than a five-point scale as it was deemed too difficult to distinguish between points in the previous rubric. In addition, user experience during the pilot phase led to the realization that there was some overlap in the note attributes assessed on the original version of the PNQI (e.g. "up-to-date" and "accurate"). There were also attributes affecting note quality that were not well captured (e.g. "patient-centeredness"). The new version of the tool assessed the following areas of each note: (1) accuracy, (2) thoughtfulness and synthesis, (3) focus and brevity, and (4) patient-centeredness, and continued to include an estimate of copied forward material.

The PNQIv2 was evaluated during the 2018-2019 academic year. Members of the study staff (RTS, CZ, DM, JM), including one neurology faculty member, one neurology fellow, one PGY3 resident and one PGY2 resident, participated in note reviews. As part of the second phase, a training document was created including example evaluations, and study staff met after pilot testing to resolve discrepancies. Three progress notes written by 10 junior residents were reviewed by the study staff with the goal of assessing the interrater reliability of the PNQIv2 tool. Junior residents again provided verbal consent to allow the study investigators to review their notes. We estimated using inter-class correlation coefficients (ICC) and $95 \%$ confidence intervals (95\% CI) using a multi-level random effects mode (Stata/SE 14.2).

\section{Results}

\section{Resident/Faculty Survey}

There were 31 respondents from the URMC department of neurology, which included 19 faculty members, 1 recent graduate of the residency program, and 12 residents (2 PGY1s, 3 PGY2s, 3 PGY3s and 4 PGY4s). $15.6 \%$ of respondents rated the neurology inpatient note quality as poor orvery poor , $50 \%$ rated the quality as neutral, and only $34.4 \%$ rated the quality as good or very good. Compared to residents, faculty generally rated progress notes as being lower in quality with only $26.3 \%$ of faculty rating them as good (and none rating the notes as very good ).

Most respondents reported that over half of progress notes contained material copied from prior notes with $46.9 \%$ indicating that $76-100 \%$ of the note was copied forward. $87.5 \%$ indicated that they believed the copy forward function created error. $48 \%$ of respondents reported that they had encountered a significant error in a progress note. Respondents identified the following factors as the most common leading to errors: inappropriate use of copied text, overuse of templated material, inclusion of redundant material, failure of notes to be up-to-date, and poorly synthesized assessments and plans.

\section{PNQI Pilot Phase}

Between October 2017 and March 2018, two members of the study staff (RTS and CZ) each reviewed 63 progress notes. These notes had been written by 12 different residents out of 33 possible residents (there was a $36 \%$ participation rate amongst the junior residents rotating through neurology). Total PNQI scores for progress notes ranged from 10 to 19 (of a possible score of 20), with a mean of 16.6 (S.D. of 2.1). Interrater reliability between the study investigators for the total PNQI scores was found to be moderate at 0.41 (95\%CI: .20-.65). Additionally, $82.5 \%$ of all the notes were estimated to contain $50 \%$ or more copied forward material. 


\section{PNQI Second Phase}

Thirty notes were reviewed between August 2018 and January 2019 by the four study investigators for a total of 120 note reviews. These notes had been written by a different group of 10 junior residents out of a possible 16 residents (representing a 63\% participation rate). Total PNQIv2 scores for progress notes ranged from 4 to 12 (of a possible score of 12), with a mean of 9.2 (SD 2). Each sub-section is scored out of 3 , and mean scores for each sub-section of the PNQIv2 were as follows: accuracy 2.6 (SD 0.6), thoughtfulness and synthesis 2.35 (SD 0.7), focused and succinct 1.95 (SD 0.7), and patient-centered 2.3 (SD 0.8). Inter-rater reliability of raters nested in residents was found to be good at 0.7 (95\% CI, .64; .75). The highest agreement was in the estimated percentage of the note that was copied forward, with $63 \%$ of notes containing at least $2 / 3$ copiedforward material. Of the PNQI subsections, inter-rater agreement was highest for "patient centeredness" and was lowest for "thoughtful and synthesized". Feedback from study staff revealed that the tool was quick and simple to use.

\section{Discussion}

Through this study, we created an evaluation tool to assess inpatient progress note quality that was simple, practical for use on the wards, and had good inter-rater reliability. In addition, the tool should be easily generalizable to other inpatient services since there is no neurology-specific language. Our first attempt showed moderate inter-rater reliability, and there was redundancy in some measures (e.g. "up-to-date" and "accurate") as well as missing information (e.g. "patient-centeredness"). Based on feedback from the pilot study, we created a second version of the note assessment tool (PNQIv2).

Our consensus was that a progress note should score at least a 9 on the PNQIv2 to be considered adequate quality. This would constitute a score of 3 for "accuracy" and at least a score of 2 on all other note attributes assessed. We recommend that a good progress note would score a 10 on the PNQIv2 whereas an excellent progress note would receive a score of 11-12. A score of 8 or less on the PNQIv2 equates topoor quality, and would be an indicator that the resident could benefit from additional education on medical documentation.

We believe that our tool has the advantage of being shorter and easier to use than previously published tools. The shorter and simpler formatting of our tool allows for note evaluations to be performed quickly and in real time. Additionally our tool uses a 3-point rubric with clear language-based descriptions rather than a 5-point Likert scale. We changed from a 5-point scale to a 3 -point scale in an effort to reduce inter-rater variability and promote consistency in scoring.

Results from the 2016 resident/faculty survey revealed that most respondents believed resident-written progress notes on the URMC inpatient neurology service were of neutral or poor quality. Evaluation of progress notes by study staff determined that the notes received an average PNQIv2 score of 9.2, and $60 \%$ of notes were determined to be at least adequate quality by receiving a PNQIv2 score of [?] 9. Although only one resident had a mean PNQIv2 score of [?] 11 for all progress notes, 6 out of the 10 residents had mean PNQIv2 scores of [?] 9 for all of their notes. It is reassuring that the majority of progress notes were considered adequate, however, our tool uncovered approximately $40 \%$ of residents who needed feedback and additional instruction on producing quality progress notes.

Our experiences with the PNQIv2 tool were promising, and as a next step, the investigators will be incorporating it into routine use for resident education. Specifically, residents will receive an electronic training guide on writing quality progress notes at the beginning of their rotation, along with weekly real-time evaluations of their notes utilizing the PNQIv2. The tool itself will serve as a source of feedback and learning for residents. Residents will be encouraged to use a progress note template that is simple with minimal amounts of pre-populated information, which will promote authorship of notes that are focused and succinct without excessive "note bloat," and will minimize inaccuracies due to copy-forward.

There are several limitations to this study. For the second phase of this study, notes from only 10 residents were reviewed to test the PNQIv2 tool. Additionally, most of the resident rotators on the neurology service were PGY1s, and the tool was not tested on senior residents. The quality of resident progress notes may 
improve as they progress through training. Only 30 progress notes were reviewed per resident for the second phase of this study, which is a relatively small number and may have led to sampling bias. In the second phase of our study, three of the four note reviewers were either neurology residents or fellows. The background and experience level of the reviewers may have affected PNQIv2 scores as faculty may grade a progress note more harshly than trainees. While we feel that the instrument should easily generalize to other services and institutions, the PNQIv2 was not tested in other hospitals or within other departments at our institution.

In conclusion, the pilot phase of our study demonstrated that there are multiple obstacles to creating a useful and reliable note assessment tool, such as finding a balance between developing a comprehensive evaluation rubric and maintaining a brief and user-friendly tool. Ultimately, the investigators developed a progress note assessment tool that was simple and practical to use on the wards, and had good inter-rater reliability. In comparison to other note assessment tools, our tool has the advantage of being shorter while still assessing key attributes of progress notes, and uses a rubric with language-based scoring descriptors rather than Likert scales. We believe that this tool has potential for widespread use in graduate medical education in evaluation of trainee documentation with the ability to teach residents to write accurate, updated, succinct, thoughtful, and patient-centered inpatient progress notes.

\section{References}

1. Obeso V, et al. Core EPAs for Entering Residency Pilot Program. Toolkits for the 13 Core Entrustable Professional Activities for Entering Residency. Association of American Medical Colleges. https://www.aamc.org/system/files/c/2/484778-epa13toolkit.pdf. 2017. Accessed December 1, 2019.

2. Wald HS, George P, Reis SP, Taylor JS. Electronic health record training in undergraduate medical education: bridging theory to practice with curricula for empowering patient- and relationship-centered care in the computerized setting. Acad Med. 2014 Mar;89(3):380-6.

3. Welcher CM, Hersh W, Takesue B, Stagg Elliott V, Hawkins RE. Barriers to Medical Students' Electronic Health Record Access Can Impede Their Preparedness for Practice. Acad Med. 2018 Jan;93(1):48-53.

4. Milano CE, Hardman JA, Plesiu A, Rdesinski RE, Biagioli FE. Simulated electronic health record (SimEHR) curriculum: teaching EHR skills and use of the EHR for disease management and prevention. Acad Med. 2014 Mar;89(3):399-403.

5. Lee WW, Alkureishi ML, Wroblewski KE, Farnan JM, Arora VM. Incorporating the human touch: piloting a curriculum for patient-centered electronic health record use. Med Educ Online. 2017;22(1):1396171.

6. Cimino JJ. Improving the electronic health record-are clinicians getting what they wished for? JAMA . 2013;309(10):991-2.

7. Edwards ST, Neri PM, Volk LA, Schiff GD, Bates DW. Association of note quality and quality of care: a cross-sectional study. BMJ Qual Saf. 2014 May;23(5):406-13.

8. Bernat JL. Ethical and quality pitfalls in electronic health records.Neurology . 2013 Mar 12;80(11):105761.

9. Stetson PD, Bakken S, Wrenn JO, Siegler EL. Assessing Electronic Note Quality Using the Physician Documentation Quality Instrument (PDQI-9). Appl Clin Inform . 2012;3(2):164-174.

10. Hammond KW, Helbig ST, Benson CC, Brathwaite-Sketoe BM. Are Electronic Medical Records Trustworthy? Observations on Copying, Pasting and Duplication. AMIA Annu Symp Proc. 2003; 2003: 269-273.

11. Versel N. "Note bloat" putting patients at risk. Every progress note shouldn't be a running blog. Healthcare IT News. https://www.healthcareitnews.com/news/note-bloat-putting-patients-risk. October 10, 2013. Accessed December 12019.

12. Rosenbloom ST, Denny JC, Xu H, Lorenzi N, Stead WW, Johnson KB. Data from clinical notes: a perspective on the tension between structure and flexible documentation. J Am Med Inform Assoc. 2011 Mar-Apr;18(2):181-6.

13. Hockstein MA, Pope SN, Donnawell K, Chavez SA, Bhat L. Emergency Medicine Residents on Electronic Medical Records: Perspectives and Advice. Cureus. 2019 Feb 7;11(2):e4027. 
14. O’Donnell HC, Kaushal R, Barrón Y, Callahan MA, Adelman RD, Siegler EL. Physicians' attitudes towards copy and pasting in electronic note writing. J Gen Intern Med. 2009 Jan;24(1):63-8.

15. Hirschtick RE. A piece of my mind. Copy-and-paste. JAMA. 2006 May 24;295(20):2335-6.

16. Gagliardi JP, Turner DA. The Electronic Health Record and Education: Rethinking Optimization. J Grad Med Educ. 2016 Jul;8(3):325-7.

17. Thielke S, Hammond K, Helbig S. Copying and pasting of examinations within the electronic medical record. Int J Med Inform. 2007 Jun;76 Suppl 1:S122-8.

18. Cohen GR, Friedman CP, Ryan AM, Richardson CR, Adler-Milstein J. Variation in Physicians' Electronic Health Record Documentation and Potential Patient Harm from That Variation. J Gen Intern Med. 2019 Nov;34(11):2355-2367.

19. Aylor M, Campbell EM, Winter C, Phillipi CA. Resident Notes in an Electronic Health Record. Clin Pediatr (Phila). 2017 Mar;56(3):257-262.

20. Pageler NM, Friedman CP, Longhurst CA. Refocusing medical education in the EMR era. JAMA. 2013 Dec 4;310(21):2249-50.

21. Otokiti A, Sideeg A, Ward P, Dongol M, Osman M, Rahaman O, Abid S. A quality improvement intervention to enhance performance and perceived confidence of new internal medicine residents. J Community Hosp Intern Med Perspect. 2018 Aug 23;8(4):182-186.

22. DeLeon S, Mothner B, Middleman A. Improving student documentation using a feedback tool. Clin Teach. 2018 Feb;15(1):48-51.

23. Sando KR, Skoy E, Bradley C, Frenzel J, Kirwin J, Urteaga E. Assessment of SOAP note evaluation tools in colleges and schools of pharmacy. Curr Pharm Teach Learn. 2017 Jul;9(4):576-584.

24. Davaridolatabadi N, Sadoughi F, Meidani Z, Shahi M. The Effect of Educational Intervention on Medical Diagnosis Recording among Residents. Acta Inform Med. 2013;21(3):173-5.

25. Sherman JJ, Johnson CD. Assessment of pharmacy students' patient care skills using case scenarios with a SOAP note grading rubric and standardized patient feedback. Curr Pharm Teach Learn. 2019 May;11(5):513-521.

26. Tinsley JA. An educational intervention to improve residents' inpatient charting. Acad Psychiatry. 2004 Summer;28(2):136-9.

27. Liang JW, Shanker VL. Education in Neurology Resident Documentation Using Payroll Simulation. J Grad Med Educ. 2017 Apr;9(2):231-236.

28. Benke JR, Lin SY, Ishman SL. Directed educational training improves coding and billing skills for residents. Int J Pediatr Otorhinolaryngol. 2013 Mar;77(3):399-401.

29. As-Sanie S, Zolnoun D, Wechter ME, Lamvu G, Tu F, Steege J. Teaching residents coding and documentation: effectiveness of a problem-oriented approach. Am J Obstet Gynecol. 2005 Nov;193(5):1790-3.

30. Patel A, Ali A, Lutfi F, Nwosu-Lheme A, Markham MJ. An Interactive Multimodality Curriculum Teaching Medicine Residents About Oncologic Documentation and Billing. MedEdPORTAL. 2018 Aug 30;14:10746.

31. Weizberg M, Cambria B, Farooqui Y, Hahn B, Dazio F, Maniago EM, Berwald N, Kass D, Ardolic B. Pilot study on documentation skills: is there adequate training in emergency medicine residency? J Emerg Med. 2011 Jun;40(6):682-6.

32. Walker KJ, Wang A, Dunlop W, Rodda H, Ben-Meir M, Staples M. The 9-Item Physician Documentation Quality Instrument (PDQI-9) score is not useful in evaluating EMR (scribe) note quality in Emergency Medicine. Appl Clin Inform. 2017 Sep 26;8(3):981-993.

33. Burke HB, Hoang A, Becher D, et al. QNOTE: an instrument for measuring the quality of EHR clinical notes. J Am Med Inform Assoc. 2014 Sep-Oct;21(5):910-6.

Acknowledgements: None

Funding: This research received no specific grant from any funding agency in the public, commercial, or not-for-profit sectors.

Declaration of Conflicts of Interest: The authors declare that there is no conflict of interest 
Tables: none

Figure legends

Figure 1: Copy of the PNQI v2 (see separate word document)

Appendices: We are attaching a copy of the faculty/resident survey as an appendex (see separate file)

\section{List of abbreviations}

EMR: electronic medical record

URMC: University of Rochester Medical Center

PGY: Post-graduate year

PDQI-9: Physician Documentation Quality Instrument - 9

PNQI: Progress Note Quality Instrument

PNQIv2: Progress Note Quality Instrument Version 2

ICC: Inter-class correlation coefficient

CI: Confidence interval

\section{Hosted file}

Progress Note Quality Instrument_v2.docx available at https://authorea.com/users/300788/ articles/430519-teaching-and-maintaining-quality-of-resident-inpatient-progress-notesdevelopment-of-the-progress-note-quality-instrument 